To be submitted to Physical Chemistry Chemical Physics (Perspective)

\title{
Double-hybrid density functionals: merging wavefunction and density approaches to get the best of both worlds
}

\author{
J. C. Sancho-García* \\ Departamento de Química Física, \\ Universidad de Alicante, E-03080 Alicante, Spain. \\ C. Adamo $^{\dagger}$ \\ Laboratoire d'Electrochimie, Chimie des Interfaces \\ et Modélisation pour l'Energie, CNRS UMR-7575, \\ Chimie ParisTech, F-75231 Paris, France \\ and \\ Institut Universitaire de France, \\ 103 Boulevard Saint Michel, F-75005 Paris, France. \\ June 10, 2013
}

\footnotetext{
*E-mail: jc.sancho@ua.es

†E-mail: carlo-adamo@chimie-paristech.fr
} 


\begin{abstract}
We review in this Perspective why and how double-hybrid density functionals have become new leading actors in the field of computational chemistry, thanks to the combination of an unprecedented accuracy together with large robustness and reliability. Similarly to their predecessors, the widely employed hybrid density functionals, they are rooted on the Adiabatic Connection Method from which they emerge in a natural way. We present recent achievements concerning applications to chemical systems of the most interest, and current extensions to deal with challenging issues such as non-covalent interactions and excitation energies. These promising methods, despite a slightly higher computational cost than other typical density-based models, are called to play a key role in the near future and can thus pave the way towards new discoveries or advances.
\end{abstract}




\section{Introduction}

John C. Slater, at the beginning of the 1950s, makes a statement which is still a highly topical issue: "Do you want to calculate it or do you want it to be accurate". That brilliant idea, to replace the exact exchange term by an approximate expression easier to calculate [1], reducing thus the computational scaling by an order of magnitude, is still feeding the field. Admittedly, the blooming of Density Functional Theory (DFT) along last decades has enormously benefited from the above audacity; a major breakthrough was the combination of exact-like and approximate exchange in a hybrid fashion together with the introduction of correlation effects through the corresponding approximate functional form [2-7]. But, who will want to go a step further by augmenting an order of magnitude the cost with respect to these hybrid exchange-correlation functionals? That (another) audacity, in pursue of the greatest possible accuracy and an unprecedented robustness, indeed leads to the development of the so-called Double-Hybrid (DH) functionals, where one combines expressions taken from ab initio theory, aimed at truly describing quantum effects such as exchange or correlation energy, with less costly density functionals, prepared to accurately mimic that description. A step further means that not only the exchange energy is hybridised but correlation energy too, which is behind the higher scaling found for the whole expression since the simplest correlated ab initio envisioned approach, perturbative or not, has a higher scaling with size than the corresponding correlation density functional. Evidently, the field has fortunately advanced so much that the combined use of numerical techniques to alleviate that extra cost gained, together with an amazing (endless) growing of world-wide computational capacities, has allowed to implement in practice that (still pioneering) idea. Of course, when a new methodology (also called tradition- 
ally model chemistry) emerges, it is always necessary to do an exhaustive benchmarking against highly accurate data, with key applications not only to known problems but to systems situated at the edge of current knowledge or for which former theories can only describe poorly the dominant effects. And this is so because Chemistry, Physics, and Materials Science have largely demonstrated an almost limitless capacity to drive wellbeing, economic growth and environmental sustainability along last decades thanks to new advances and achievements. If we want to continue into this direction, and thus to manipulate the matter at a submicroscopic size to create tailored materials paving the way towards new discoveries or technologies with a vast range of applications, Quantum and Computational Chemistry are called to play a key role, now and ever. We welcome you to this fascinating adventure.

\section{Theoretical framework}

\subsection{Generalities: ACM and associated functionals}

One of the most fascinating fields in DFT is the determination of new exchange-correlation functionals based on well-defined physical models, that is functionals that do not contain any parameter fitted to external (experimental or theoretical) data. Such parameter-free Density Functional Approximations (DFAs), undoubtedly represent a very challenging playground which, in a parallel with the harsh world of mountaineering, could be defined as "the cleanest line on the steepest part of the face" [8]. Indeed these DFAs not only are based on a solid physics ground ("the cleanest line"), but they have the ambition to point towards the so-called "chemical accuracy" ("the steepest part of the face"), often offering numerical performances comparable to those obtained with functionals containing a (large) number of 
fitted parameters. DFT provides a rigorous framework to these DFAs and several models have been used to develop functionals based on Local Density and Generalized Gradient Approximations (LDA and GGA, respectively), see for instance references [9-13]. Among others, the model developed by Perdew, Burke and Ernzerhof (PBE) [14] is undoubtedly the most representative functional of such a family, and it clearly shows how interesting numerical performances can be obtained imposing some of the theoretical limits of the ideal functional. As a matter of fact, the PBE functional generally provides results for a large number of properties and systems at least as good as the most-used parameterized approaches, such as BLYP $[15,16]$, and sometimes even better.

Still however the performances of such DFAs are far from the required accuracy for a large number of properties and their progress is mandatory. Once again, the rigorous formal framework of DFT provided the way out. In particular, the Adiabatic Connection Model (ACM) [17] allows for defining the exchange-correlation contribution, $E_{x c}$, to the total Kohn-Sham (KS) energy as:

$$
E_{x c}[\rho]=\int_{0}^{1} E_{x c, \lambda}[\rho] d \lambda
$$

here

$$
E_{x c, \lambda}[\rho]=\left\langle\Psi_{\lambda}\left|\hat{V}_{e e}\right| \Psi_{\lambda}\right\rangle-\frac{1}{2} \iint \frac{\rho(\mathbf{r}) \rho\left(\mathbf{r}^{\prime}\right)}{\left|\mathbf{r}-\mathbf{r}^{\prime}\right|} d \mathbf{r}^{\prime} d \mathbf{r}
$$

Note that $\Psi_{\lambda}$ is the KS wave function minimizing

$$
\left\langle\Psi_{\lambda}\left|\hat{T}+\lambda \hat{V}_{e e}\right| \Psi_{\lambda}\right\rangle
$$

and $\hat{V}_{e e}$ is the electron-electron interaction term. The variable $\lambda$ allows for connecting the non-interacting system (reference system, $\lambda=0$ ) with the real interacting one (physical system, $\lambda=1$ ), assuming that the external 
potential is adjusted to hold the electron density $\rho$ fixed at its value for $\lambda=1$. It is also usually supposed that the system is at the best described by a given DFA at $\lambda=1$, that is:

$$
E_{x c, \lambda=1}=E_{x c}^{\mathrm{DFA}}
$$

At $\lambda=0$ the KS noninteracting system should be recovered, so that:

$$
E_{x c, \lambda=0}=E_{x}
$$

$E_{x}$ being the Hartree-Fock (HF) exchange term but computed with the KS orbitals; i.e., a kind of EXact-like Exchange (EXX). A simple two-point approximation of the integral given by Eq. (1) gives:

$$
E_{x c} \approx\left(E_{x c, \lambda=0}+E_{x c, \lambda=1}\right)=E_{x}^{\mathrm{EXX}}+E_{x c}^{\mathrm{DFA}} .
$$

Actually, the Becke Half\&Half approach is expressed in such a way and it represents the first hybrid functional mixing EXX and DFA in a notapproximated way [2]. In the same line, the popular B3LYP functional $[3,18,19]$ can be then seen as an empirical approximation of integral (1), giving thus:

$$
E_{x c, \lambda}^{\mathrm{B} 3 \mathrm{LYP}}=E_{x c}^{\mathrm{LDA}}+a_{0}\left(E_{x}^{\mathrm{EXX}}-E_{x}^{\mathrm{S}}\right)+a_{x}\left(E_{x}^{\mathrm{B}}-E_{x}^{\mathrm{S}}\right)+a_{c}\left(E_{x}^{\mathrm{LYP}}-E_{c}^{\mathrm{VWN}}\right)
$$

It is worth to mention than the three parameters ruling the EXX and DFA contributions in B3LYP, $a_{0}, a_{x}$ and $a_{c}$, are fitted on the G2 dataset [3].

Albeit the dependence of the universal functional upon the coupling constant $\lambda$ is not known, some hypothesis have been done in literature [20-23]. For instance, Perdew and co-workers assumed that [4]:

$$
E_{x c, \lambda}^{h y b r i d}=E_{x c}^{\mathrm{DFA}}+\left(E_{x}^{\mathrm{EXX}}-E_{x}^{\mathrm{DFA}}\right)(1-\lambda)^{n-1},
$$


where the integer $n$ determines how fast the correction to DFA (second term of equation 8) vanishes for $\lambda \rightarrow 1$. Upon integration, we arrive at the following expression:

$$
E_{x c}^{\text {hybrid }}=\int_{0}^{1} E_{x c, \lambda}^{h y b r i d} d \lambda=E_{x c}^{\mathrm{DFA}}+\frac{1}{n}\left(E_{x}^{\mathrm{EXX}}-E_{x}^{\mathrm{DFA}}\right) .
$$

It has been then argued $[4,5]$, using results from perturbation theory, than $n=4$ is the optimal value for a realistic description of the chemical systems. When the PBE exchange-functional is casted, the PBE0 approach is thus correspondingly obtained $[6,7]$ :

$$
E_{x c}^{\mathrm{PBE} 0}=E_{x c}^{\mathrm{PBE}}+\frac{1}{4}\left(E_{x}^{\mathrm{EXX}}-E_{x}^{\mathrm{PBE}}\right) .
$$

Such a functional can be considered as the first hybrid DFA completely parameter-free, and it has been proven to be comparable in accuracy with B3LYP and sometimes it is even better (e.g. excited state properties) [24-28]. It is worth to mention that very recently a different $\lambda$-dependence has been proposed, leading to a different integer for $n[29,30]$.

In the same spirit, the ACM provides also a formal framework for the development of new DH functionals, containing a correlation contribution evaluated with a second-order perturbation theory, an approach firstly evidenced by Ernzerhof almost fifteen years ago [31]. Let us to write the Hamiltonian of our system with the explicit dependence upon the parameter $\lambda$ :

$$
\hat{H}_{\lambda}=\hat{T}+\hat{V}_{e x t}+\lambda \hat{V}_{e e}+\hat{V}_{H x c}^{\lambda}
$$

where $\hat{T}$ represents the kinetic energy operator, $\hat{V}_{e x t}$ is the external potential, $\hat{V}_{e e}$ is the electron-electron interactions and $\hat{V}_{H x c}$ is the operator containing the exchange and correlation contributions as well as the Hartree potential 
operator. In the density-scaled one-parameter hybrid approximation [32], the total energy is then defined by minimization of the expression:

$$
E^{\lambda}=\min _{\phi}\left\{\left\langle\Psi\left|\hat{T}+\hat{V}_{e x t}+\lambda \hat{V}_{e e}\right| \Psi\right\rangle+E_{H x c}^{\lambda}\right\}
$$

The term $E_{H x c}^{\lambda}$ represents the difference between the standard KS exchangecorrelation energy and its $\lambda$-dependent counterpart and generates the corresponding operator of equation (11). The dependence on $\lambda$ of the different terms entering into $E_{H x c}^{\lambda}$ is not the same [33]:

$$
\begin{aligned}
E_{H}^{\lambda}[\rho] & =(1-\lambda) E_{H}[\rho] \\
E_{x}^{\lambda}[\rho] & =(1-\lambda) E_{x}[\rho] \\
E_{c}^{\lambda}[\rho] & =E_{c}[\rho]-\lambda^{2} E_{c}\left[\rho_{\frac{1}{\lambda}}\right],
\end{aligned}
$$

where $E_{c}[\rho]$ is the KS correlation energy, $E_{c}^{\lambda}[\rho]$ is the correlation corresponding to the Hamiltonian operator of equation (11) and $\rho_{\frac{1}{\lambda}}=\left(\frac{1}{\lambda}\right)^{3} \rho\left(\frac{\mathbf{r}}{\lambda}\right)$ is the scaled density. If the wave function $\phi$ is expressed as a single determinant, the energy in equation (12) can be written as:

$$
E^{\lambda}=\left\langle\phi\left|\hat{T}+\hat{V}_{e x t}\right| \phi\right\rangle+E_{H}[\rho]+\lambda E_{x}^{\mathrm{EXX}}[\phi]+(1-\lambda) E_{x}[\rho]+E_{c}[\rho]-\lambda^{2} E_{c}\left[\rho_{\frac{1}{\lambda}}\right]
$$

where

$$
E_{x c}^{\lambda}=\lambda E_{x c}^{\mathrm{EXX}}[\phi]+(1-\lambda) E_{x}[\rho]+E_{c}[\rho]-\lambda^{2} E_{c}\left[\rho_{\frac{1}{\lambda}}\right] .
$$

This latter contribution is close to those obtained for hybrid functionals such as PBE0 or B3LYP (see equations 7 and 10), but the scaled functional $E_{c}\left[\rho_{\frac{1}{\lambda}}\right]$ is weighted by $\lambda^{2}$. What is still missing in this expression is the contribution coming from the $V_{e e}$ term, which can be expressed using a second-order Perturbation Theory (PT2):

$$
E_{x c}^{\lambda}=\lambda E_{x}^{\mathrm{EXX}}[\phi]+(1-\lambda) E_{x}[\rho]+E_{c}[\rho]-\lambda^{2} E_{c}\left[\rho_{\frac{1}{\lambda}}\right]+\lambda^{2} E_{c}^{\mathrm{PT} 2}
$$


Here the difficulty is represented by the scaled correlation contribution $E_{c}\left[\rho_{\frac{1}{\lambda}}\right]$, and conveniently illustrated in Ref. [32]; however, an hypothesis on the $\lambda$ dependency should be introduced for computational purposes. Indeed, it can be assumed that the correlation does not scale with $\lambda$, as in the $1 \mathrm{DH}-$ PBE model [32], or that the scaling is simply linear [34]. However, this term tends to the second-order Görling-Levy (GL2) correlation energy in the weak-interaction limit (and for non-degenerate KS systems) [35]:

$$
\lim _{\lambda \mapsto 0} E_{c}\left[\rho_{\frac{1}{\lambda}}\right]=E_{c}^{\mathrm{GL} 2}
$$

The GL2 energy, derived from a second-order truncation of the couplingconstant perturbation expansion in power of $\lambda$, is:

$E_{c}^{\mathrm{GL} 2}=\frac{1}{4} \sum_{i j} \sum_{a b} \frac{\left|\left\langle\varphi_{i} \varphi_{j}\left|\hat{V}_{e e}\right| \varphi_{a} \varphi_{b}\right\rangle\right|^{2}}{\varepsilon_{a}+\varepsilon_{b}-\varepsilon_{i}-\varepsilon_{j}}-\sum_{i a} \frac{\left|\left\langle\varphi_{i}\left|v_{x}-f\right| \varphi_{a b}\right\rangle\right|^{2}}{\varepsilon_{a}-\varepsilon_{i}}=E_{c}^{\mathrm{PT} 2}+E_{c}^{\Delta \mathrm{EXX}}$,

where the indices $i$ and $j$ run over the occupied KS orbitals and $a$ and $b$ over the unoccupied ones. The $\varepsilon_{i}$ are the energies of the KS orbitals, $f$ is the non-local EXX exchange potential and $v_{x}$ is the local KS exchange potential. The $E_{c}^{\Delta \mathrm{EXX}}$ contribution is usually negligible [36], so that

$$
E_{c}^{\mathrm{GL} 2} \approx E_{c}^{\mathrm{PT} 2}
$$

being $E_{c}^{\mathrm{PT} 2}$ the usual expression (i.e., Møller-Plesset-like) for correlation energy arising from perturbation theory truncated at second-order. If a linear interpolation is then used [34], the scaled correlation contribution can be expressed as:

$$
E_{c}\left[\rho_{\frac{1}{\lambda}}\right]=(1-\lambda) E_{c}^{\mathrm{PT} 2}+\lambda E_{c}
$$

Substituting equation (22) into (18) an expression for One-Parameter Double Hybrid (OP-DH) is obtained:

$$
E_{x c}^{\lambda}=\lambda E_{x}^{\mathrm{EXX}}[\phi]+(1-\lambda) E_{x}[\rho]+\left(1-\lambda^{3}\right) E_{c}[\rho]+\lambda^{3} E_{c}^{\mathrm{PT} 2} .
$$


Still parameter $\lambda$ has to be determined. A possible way is fitting to a given dataset or, alternatively, it could be determined by casting equation (23) in the ACM formula (equation 1) and then evaluating the integral with a twopoint formula: this leads to $\lambda=\frac{1}{2}$. If, as done for PBE0, the PBE functional is considered, the PBE0-DH model is then obtained [37]:

$$
E_{x c}^{\mathrm{PBE} 0-\mathrm{DH}}=\frac{1}{2}\left(E_{x}^{\mathrm{EXX}}+E_{x}^{\mathrm{PBE}}[\rho]\right)+\frac{1}{2}\left(\frac{7}{4} E_{c}^{\mathrm{PBE}}[\rho]+\frac{1}{4} E_{c}^{\mathrm{PT} 2}\right)
$$

It is noteworthy that such a model adds two more constraints to those already used for PBE, namely a linear dependence on $\lambda$ and the respect of the GL limit. Such increased number of theoretical fundamentals does not affect the numerical performances of PBE0-DH: the numerical tests (see below) clearly show that PBE0-DH is systematically better than PBE0. Therefore the increase of the theoretical basics is even beneficial for functionals' accuracy. Equation (23) is very close to the traditional multi-parameters double hybrids such as, for instance, B2-PLYP [35]:

$$
E_{x c}^{\mathrm{DH}}=a_{x} E_{x}^{\mathrm{EXX}}+\left(1-a_{x}\right) E_{x}^{\mathrm{B}}+\left(1-a_{c}\right) E_{c}^{\mathrm{LYP}}+a_{c} E_{c}^{\mathrm{PT} 2} .
$$

However, while equation (25) contains a pair of (possibly coupled) parameters (which have to be added to those already present in the underlying $\mathrm{B}$ and LYP functionals) equation (23) contains only one parameter, which, as discussed, could be determined a priori. Finally it should be remarked the strong connection between DH and Range Separated Approaches (RSH). In these latter, the two-electron Coulomb interaction, $1 / r_{12}$, is separated into a long and a short part [38]. The short-range is usually treated at DFT level, while long-range part can be modeled using a wave-function approach, such as HF [39], MP2 [40] or even more refined post-HF schemes [40-42]. In such RSHs, the parameter $\mu$ controls the separation between the two parts: for $\mu=0$ the long-range part vanishes so that a full DFT approach is recovered, 
while for $\mu \rightarrow \infty$ the long-range part dominates [43]. Such an approach has been applied to the evaluation of long-range correlation corrections using a perturbation approach where total energy (cf. Equation 12) explicitly depends upon $\lambda$ and $\mu$ [40]. This approach represents therefore a kind of generalization of the double hybrid models.

So at the end, two families can be defined starting from ACM: that originating from BLYP and including B3LYP and B2-PLYP and the other comprising PBE, PBE0 and PBE0-DH. The first is, of course, semi-empirical in nature, albeit only few parameters are used, while the second is parameterfree but it rests on some (still arbitrary) assumptions. As it will be showed in the following, the two series provide very close results, showing comparable accuracies for a number of properties. Between them, it should be mentioned the XYG3 model [44,45], a semi-empirical approach to the DH problem, which is nevertheless based on a linear $\lambda$-dependence of the functional (as Becke's Half\&Half) and takes somehow into account the GL limit (equation 19), as well as some variants as the PBE0-2 model [46].

\subsection{Construction of empirical functionals}

Pioneering composite methods named Multi-Coefficient (MC) protocols $[47,48]$ can be used to rationalize the empirical route intensively followed in last years. These MC methods combine different total energies, be them $a b$ initio or DFT, as a function of two parameters, $c_{1}$ and $c_{2}$, which need to be fitted against a well-defined training set. A general envisioned expression for this family of methods might be:

$$
E^{\mathrm{MC}}=c_{2}\left[E^{\mathrm{HF}}+c_{1}\left(E^{\mathrm{MP} 2}-E^{\mathrm{HF}}\right)\right]+\left(1-c_{2}\right) E[\rho],
$$


where $E^{\mathrm{HF}}, E^{\mathrm{MP} 2}$, and $E[\rho]$ are Hartree-Fock, (Møller-Plesset) perturbation theory up to second-order, and DFT-based total energies, respectively. Successful applications of these composite methods have been reported repeatedly in the literature [49-51]. If we assume enough similarities between one-electron orbitals arising from separate solutions of the Hartree-Fock or Kohn-Sham self-consistent equations [52], and then we neglect every kinetic or potential energy differences between corresponding one-particle mean values, the MC-based exchange-correlation energy might be expressed as:

$$
E_{x c}^{\mathrm{MC}}=c_{2} E_{x}^{\mathrm{EXX}}+c_{1} c_{2} E_{c}^{\mathrm{PT} 2}+\left(1-c_{2}\right) E_{x c}[\rho],
$$

which retains the form of a double-hybrid functional and depends, in a natural fashion, from the particular form given to $E_{x c}[\rho]$ together with the original values for $c_{1}$ and $c_{2}$; being now $E_{x}^{\mathrm{EXX}}$ again a kind of EXact-like Exchange (EXX) energy still to be defined. Note that the final values obtained for $c_{1}$ and $c_{2}$ are at the same time related to some exchange-correlation kernel due to the target function used for their parameterisation. This feature underlines the existence of several possibilities according to literature (i.e., admittedly, as a matter of taste): choosing for $E_{x c}[\rho]$ a pure or a hybrid form will change the underlying proportions between the terms. It seems thus that there is enough justification (pragmatically speaking) to simply optimise the coefficients multiplying each term according to modern parameterisation strategies, as it has been done routinely for hybrid functionals in last decades. One can thus easily reexpress equation (27) as a function of the following weights $\left(\mathrm{w}_{i}\right)$,

$$
E_{x c}^{\mathrm{DH}}=\mathrm{w}_{\mathrm{EXX}} E_{x}^{\mathrm{EXX}}+\left(1-\mathrm{w}_{\mathrm{EXX}}\right) E_{x}[\rho]+\mathrm{w}_{\mathrm{PT} 2} E_{c}^{\mathrm{PT} 2}+\mathrm{w}_{\mathrm{c}} E_{c}[\rho],
$$

noting that $\mathrm{w}_{\mathrm{PT} 2}+\mathrm{w}_{\mathrm{c}} \neq 1$ necessarily, although one can normally constraint the final number of independent parameters by imposing $\mathrm{w}_{\mathrm{c}}=\left(1-\mathrm{w}_{\mathrm{PT} 2}\right)$. 
Notwithstanding this, the large popularisation of these (semiempirical) methods was possibly due to the seminal paper by Grimme [35] presenting the B2-PLYP model, and the subsequent mPW2-PLYP variant [53], together with some further technical advances as truly analytical derivatives [54], which allows rapid evaluation of geometries, or density-fitting techniques $[55,56]$, which alleviates the (extra) associated computational cost. Table 1 gathers a summary of existing expression within this category [57-68]; broadly speaking, they differ in: (i) the kernel used to represent the exchange-correlation effects (i.e., B2-PLYP vs. mPW2-PLYP); (ii) the training set used to obtain the value of the $\left\{\mathrm{w}_{\mathrm{EXX}}, \mathrm{w}_{\mathrm{PT} 2}\right\}$ weights, which is also related to the nature of the target set and the purpose of the parameterization (i.e., B2-PLYP vs. B2K-PLYP); (iii) the relaxation of some of the constraints $\left(1-\mathrm{w}_{i}\right)$ relating the ab initio and DFT-based counterparts (i.e., B2 $\pi$-PLYP vs. B2-PLYP); and (iv) the origin of the orbitals introduced into the perturbative term $E_{c}^{\mathrm{PT} 2}$, if they arise from the the self-consistent solution of the ad hoc unperturbed KS equations or if they come from a complete solution such as B3LYP (i.e., B2-PLYP vs. XYG3). Upon inspection of Table 1 , it is readily seen how the weight of EXX is always considerably high, compared with the moderate weight for the perturbative term or with corresponding weight in hybrid models, and that a recently highlighted [69] condition such as $\mathrm{w}_{\mathrm{PT} 2} \leq \mathrm{w}_{\mathrm{Exx}}{ }^{2}$, is almost invariably fulfilled independently of the final composition of these DH forms. These features are expected to largely drive the successful performance of the methods studied so far, as we will see next.

One can also easily verify how the artillery of modern quantum chemistry 
has also successfully impregnated the development of these methods. As a matter of illustration, a variant of canonical MP2, that scales differently the contribution to correlation energy arising from same- or opposite-spin pairs, was recently introduced (the Spin-Component-Scaled MP2 [70])to largely remedy the intrinsic deficiencies of the original MP2 method. Actually, commonly used correlation functionals mainly introduce short-range dynamical correlation effects [71,72], and thus partly neglect correlations (i.e., left-right) between parallel-spin pairs, which leaves this responsibility to the MP2-type term of double-hybrid functionals. Therefore, due to this unbalanced treatment of correlation effects, some recent expressions (e.g. DSD-BLYP) assign different weights to same- or opposite-spin coefficients, see Table 1, which are necessarily of an empirical nature. Furthermore, the decomposition of electron-electron interactions into short- and long-range terms, which also copes with the intrinsic deficiencies of any method to accurately deal with exchange-correlation effects at any interelectronic separation regime, has also encountered successful applications [73-75] in this framework (e.g. $\omega$ B97X-2 or lrc-XYG3).

\subsection{Extensions}

\subsubsection{Non-covalent interactions}

Mutually polarizable electronic clouds, even if they just weakly overlap, are known to reciprocally influence each other through instantaneous creation/annhilitation of dipole-dipole interactions. This stabilising effect due to locally induced dipole moments will thus appear whenever polarizable electronic clouds are present in two spatially separated but interacting sub-

systems, be them intramolecular and (more notoriously) intermolecular frag- 
ments [76]. The physics behind these interactions would need a kernel able to capture information simultaneously from two separate spatial points, situated at $\mathbf{r}$ and $\mathbf{r}^{\prime}$, to adequately calculate this long-range dispersion energy [77]. This is one of the reasons why local or semi-local functionals completely fail due to the short-sighted treatment of matter imposed by their dependence on the density $(\rho)$ and its gradient $(\nabla \rho)$ on $\mathbf{r}$ exclusively. The MP2-type term, even if it is normally low-weighted $\left(\mathrm{w}_{\mathrm{PT} 2} \leq 0.5\right)$, can partly describe these interactions [78] and situates thus double-hybrid functionals in a better position with respect to standard (pure or hybrid) functionals for the treatment of these effects. For instance, application (def2-QZVP basis set) of the hierarchy formed by PBE, PBE0 and PBE0-DH methods to the NCB31 data set of non-covalent interactions [79], comprising a large set (hydrogen bonding dimers, charge transfer, dipole-dipole interactions, or $\pi$ - $\pi$ complexes) of weak interactions, provides a global deviation of $1.1,0.7$, and $0.5 \mathrm{kcal} / \mathrm{mol}$, respectively. Furthermore, we also note that the XGY3 model behaves excellently for the calculation of potential energy curves along the dissociation coordinates of weakly bound pairs of molecules like pyridine or benzene dimers [80].

Nonetheless, besides more complex formalisms as adiabatic-connection fluctuation-dissipation theorem [81] or the random-phase approximation [82], practical schemes introduce semi-classically (at no extra cost) the missing dispersion energy $\left(E_{\mathrm{Dx}}\right)$ into the computational treatment by means of a function like [83-85]:

$$
E_{\mathrm{D} 3}=-\sum_{B>A}^{N} \sum_{n=6,8} s_{n} \frac{C_{n}^{A B}}{R_{A B}^{n}} f_{n}\left(R_{A B}, s_{r, n}\right),
$$

which runs over all atom-atom $(A-B)$ pairs of the chemical system and depends on $C_{n}^{A B}$ (nth-order interatomic dispersion coefficients) and $R_{A B}$ (distance between the two atoms involved), also relying on a damping function 
$f_{n}\left(R_{A B}\right)$ switching from the infinite separate limit to shorter distances close to the binding region, avoiding at the same time singularities for small $R_{A B}$ values [86]. Note that a set of parameters $\left(s_{n}\right.$ and $\left.s_{r, n}\right)$ is needed for each functional according to their different nature (i.e., the $\mathrm{w}_{\mathrm{PT} 2}$ values). The expression can be further simplified, to keep everything as simple as possible, by saving only the first term as a function of only one parameter $\left(s_{6}\right)$ :

$$
E_{\mathrm{D} 2}=-s_{6} \sum_{B>A}^{N} \frac{C_{6}^{A B}}{R_{A B}^{6}} f\left(R_{A B}\right),
$$

which would allow a preliminary comparison among methods. It the above outlined hypothesis was true, $s_{6}$ should decrease along the hierarchy of methods being necessarily lower for the double-hybrid functionals. We will select for this assessment the widely used database called S22 [87-89], which includes most typical non-covalent interactions, such as hydrogen bonds, dispersion, and mixed electrostatic-dispersion, in small- to medium-size dimers comprising aromatic and heteroaromatic compounds, and for which nearlyexact binding energies are available. Table 2 presents the values found in literature $[35,58,90,91]$ for the $s_{6}$ parameter, as well as the Mean Unsigned Error (MUE) obtained for the S22 database; note that the large basis sets used are close enough to the asymptotic limit and thus prevent any error due to basis sets incompleteness. Perusing Table 2 shows clearly how this parameterisation works well with all considered functionals, with $s_{6}$ consistently decreasing along the hierarchy of methods (i.e., PBE, PBE0, PBE0-DH, on one hand, and BLYP, B3LYP, B2-PLYP, on the other hand). Note how the sum $\mathrm{w}_{\mathrm{PT} 2}+s_{6}$ remains lower than 1.0 due to the well-known overestimation of dispersion interactions by the MP2-type term. Note also that double-hybrid functionals are, not unexpectedly, particularly successful, providing errors below $0.5 \mathrm{kcal} / \mathrm{mol}$ for this set of challenging systems. This accuracy is preserved in other appealing and related studies [92-95] which 
drops optimism about the theoretical justification and existing protocols used for their development. The best way to afford the optimisation procedure (if the dispersion energy is added on top of the total DFT-based energy or if every weight, or even the parameters entering into the formulation itself of the underlying exchange-correlation functionals, are simultaneously optimised together with the dispersion correction) or the possible coupling of a truly non-local term (depending on both $\mathbf{r}$ and $\mathbf{r}^{\prime}$ ) to the local correlation functional, concomitantly with the MP2-type term, is also under investigation.

\subsubsection{Excited-states treatment}

Double-hybrid density functionals can be also applied to excited electronic states [96] with much expected success. Again, non-local correlation effects introduced through the MP2-type correction together with a large weight of EXX-like exchange, can hopefully help to overcome the limitations commonly found for routine Time-Dependent (TD-) DFT calculations. As long as the excited state possesses substantial charge-transfer, double-excitation or multiplet nature, TD-DFT is known to fail in these situations, lying far from the desired "chemical accuracy" for excited states (accuracy below $0.1 \mathrm{eV}$ with respect to benchmark results). It is also fair to notice that the large amount of EXX is not enough to improve the results, without an explicit inclusion of the MP2-type contribution. This is approximately done by assuming that the final excitation energy $\left(\Omega_{\mathrm{DH}}\right)$ is given by:

$$
\Omega_{\mathrm{DH}}=\Omega+\mathrm{w}_{\mathrm{PT} 2} \Delta_{(\mathrm{D})},
$$

where $\Omega$ is the excitation energy provided by a functional discarding the perturbative term, a global hybrid composed ad hoc along the treatment, and 
$\Delta_{(\mathrm{D})}$ a correction based on the CIS(D) method [97]. Note that this correction only improves excitation energies, without correcting other excitation properties (e.g., transition moments). We can compare again the accuracy along the hierarchy of methods (i.e., BLYP, B3LYP, B2-PLYP) but now on computation (def2-TZVPP basis set) of singlet-singlet excitation energies for large organic dyes (chromophores 1-12, see Figure 1) constituting a well-rooted and recently benchmarked set $[98,99]$. Whereas BLYP provides an unacceptable MUE of $0.5 \mathrm{eV}$, B3LYP improves it to $0.3 \mathrm{eV}$, and B2-PLYP (or B2GPPLYP) still reduces its value to $0.2 \mathrm{eV}$, becoming certainly competitive with other highly sophisticated methods [100]. Furthermore, the same degree of accuracy is found for singlet-triplet transitions and for excited states of polycyclic aromatic hydrocarbons of varying shape and topology [96], including a balanced description of low-lying excited-states for polyenes [101]. It seems thus that the accuracy mirrors the hierarchy of methods for both groundand excited-state properties, which is rather heart-warming and helps to reconcile theory with experiments even more. Concerning open issues for the treatment of excited states, we note that the same functional is used for both ground- and excited-states, as done normally with hybrid models; however, the $\Delta_{(\mathrm{D})}$ correction really makes a difference here, and it is scaled by the same weight in both states. Additionally, the orbitals arising from the ground-state self-consistent solution, which are normally obtained after discarding the non-local correlation part, are inserting into the derived expressions for excited-states too without analyzing in depth their shape and values. 


\subsection{Further relevant issues}

\subsubsection{Scaling and basis sets convergence}

The bottleneck for large real-world applications of double-hybrid functionals is undoubtedly the perturbative term due to its formal $O\left(N^{5}\right)$ scaling with the size of the system $(N)$. This fact was indeed early recognised for the MC-based methods, but the large accuracy of modern approaches have prompted more and more applications to larger systems, which might be somehow limited due to this scaling. Actually, the introduction of doublehybrid functionals (e.g. XYGJ-OS, PTPSS or B2OS-P3LYP) having contributions to the perturbative energy only from pairs of Opposite-Spin (OS) is largely motivated by the reduction of this scaling to $O\left(N^{4}\right)$, under proper transformation algorithms $[102,103]$. Besides this, the basis set convergence of double-hybrid calculations is expected to be slower than that for parent hybrid methods, with more difficulties to reach the asymptotic regime; we also mention the use of the explicitly correlated F12 approach for the perturbative term in an attempt to speed up this convergence [104]. Furthermore, due to the different convergence patterns experienced by DFT and MP2-type terms, standard extrapolation techniques $[105,106]$ needs to be separately applied: whereas any triple- $\xi$ level such as cc-pVTZ or def2-TZVP basis sets might be enough to reach fairly accurate results, with an accuracy below 1 $\mathrm{kcal} / \mathrm{mol}$ with respect to the basis sets limit, in standard DFT calculations, however, the same level of accuracy for double-hybrid functionals would need the corresponding 'one angular momentum' extension, i.e. cc-pVQZ or def2QZVP, to leave behind any error other than that due to the intrinsic form of the functional. This double convergence might dramatically manifest in describing non-covalent interactions, for which the basis set incompleteness will particularly affect the MP2-type term if it is not properly taken into ac- 
count. An additional issue is how transferable might be the weights of each ingredient according to the basis set used in their development. The basis set dependence of XYG3 was recently examined in detail [107], starting from the basis functions used by default in the development of the method, to infer variations of up to few $\mathrm{kcal} / \mathrm{mol}$ in the MUE of few databases including heats of formation, bond dissociation enthalpies or reaction barrier heights, upon changes to smaller basis sets. This fact has even prompted the authors to develop variants (XYG3o or XYG3s) $[108,109]$ to minimise this collateral effect and to implicitly account thus for basis set incompleteness. Finally, the different convergence pattern experienced by every term of the expression, typically $n^{-3}$ for the correlation energy of the MP2-type term and $n^{-5}$ (or exponential) for the rest of the total energy, where $n$ is associated to the highest angular momentum value of the basis sets employed, might be used to find an optimum $n^{-\alpha}$ function to estimate final results at the asymptotic regime in current calculations, or to develop reliable extrapolation formulas [110-112] taken into account that the convergence of total energy follows normally the slow component. More systematic investigations about basis set effects and convergence will be thus acknowledged.

\subsubsection{Self-interaction error}

The Self-Interaction Error (SIE) of common density functionals [113], the spurious self-interaction of an electron with itself, is expected to affect double-hybrid functionals too mostly through the exchange term. This issue is known to have particular importance when charged species are involved and/or when a transition from delocalized to localized states is expected to drive the results [114-119]. Using the database SIE11, which forms part of 
the recently launched general benchmark databases called GMTKN30 [120] and its predecessor GMTKN24 [121], comprising a set of reaction energies extremely prone to this generalised drawback, we have recently evaluated the performance of some double-hybrid forms compared to older hybrid expressions. Note that the geometries and the basis set (def2-QZVP) employed readily allows the adequate comparison between the methods in all cases. Figure 2 shows the evolution of the MUE for the pair of families PBE, PBE0, PBE0-DH, on one hand, and BLYP, B3LYP, B2-PLYP, B2GP-PLYP, on the other hand. The results evolve linearly with the value of $\mathrm{w}_{\mathrm{Exx}}$, almost independently of the form and weight of correlation terms, within each family. We remind that exact exchange is, by definition, SIE-free, which clearly helps to explain the trend found. Unless otherwise forced in future developments, neither of these expressions is one-electron SIE-free yet, the essence of selfinteraction, defined for one-electron systems having $\int d \mathbf{r} \rho_{\alpha}=1$ and $\rho_{\beta}=0$, as the conditions: (i) $E_{x}\left[\rho_{\alpha}, 0\right]=-J\left[\rho_{\alpha}\right]$; and (ii) $E_{c}\left[\rho_{i}\right]=0$. However, the B2-PLYP calculated (def2-QZVP basis set) SIE for Hydrogen atom is 0.74 $\mathrm{kcal} / \mathrm{mol}$, despite being $E_{c}[\rho]=0$ in this (LYP) case. Application now of PBE0-DH (def2-QZVP basis set) translates to twice that error, mainly due to the fact that $E_{c}[\rho] \neq 0$ in this $(\mathrm{PBE})$ case. These results clearly illustrate the (expectedly) large role played by the SIE in common applications and the existing difficulties to cope efficiently with it.

\section{Selected applications to chemical thermo- dynamics, reactivity, and materials science}

Before going into greater details, it is normally advised to present first the results for general main group thermochemistry, kinetics, and non-covalent 
interactions, which is best done through analysing the performance of $\mathrm{DH}$ forms for the GMTKN30 database. Herein, a short summary is presented; the interested readers can consult the original work [120]. The values shown in Table 3 are based on calculations with the (aug-)def2-QZVP basis sets, all of them including the -D3 correction for non-covalency effects, and drops a clear take-home-message: DH functionals clearly outperform all other developed models. Their robustness is also largely demonstrated since all of them have a Weighted Total MUE (WTMUE) lying in the range $1.5-2.5 \mathrm{kcal} / \mathrm{mol}$, which is 2-3 times lower than their corresponding ancestors. No other family of functionals behaves so accurately for such a kind of intensive benchmarking and, although their basis set dependence is higher than for other functionals, the price to be paid is well-invested. Various subsets of results are presented next as a motivation for the use of $\mathrm{DH}$ forms in challenging situations:

1. Isomerization reactions of large organic molecules (ISOL24/11 database); for which B2-PLYP-D3 and B2GP-PLYP-D3 levels give a MUE of 2.9 and $1.4 \mathrm{kcal} / \mathrm{mol}$, respectively, compared to a B3LYP-D3 value of 9.0 $\mathrm{kcal} / \mathrm{mol}[122]$.

2. Isodesmic bond separation reactions of saturated hydrocarbons (BSR36 database); for which B2-PLYP-D3 and B2GP-PLYP-D3 lead to a MUE of 2.4 and $1.7 \mathrm{kcal} / \mathrm{mol}$, respectively, compared to a B3LYP-D3 value of $5.9 \mathrm{kcal} / \mathrm{mol}[123]$.

3. Isodesmic reaction energies of $n$-alkanes to ethane; for which the errors systematically become larger upon increasing the size of the alkane. Only the DH forms employed (B2-PLYP-D3 and B2GP-PLYP-D3) are able to approach the desired accuracy, with errors less than $2 \mathrm{kcal} / \mathrm{mol}$ [124]. 
4. Activation (forward and backward) barriers for hydrogen and heavy atom transfer, nucleophilic substitutions or unimolecular association reactions (DBH24/08 database); for which PBE0-DH drops a MUE of $1.6 \mathrm{kcal} / \mathrm{mol}$, substantially lower than PBE0 $(4.0 \mathrm{kcal} / \mathrm{mol})$ or PBE $(8.2$ $\mathrm{kcal} / \mathrm{mol}$ ) [37]. This accuracy is preserved for intra- and intermolecular proton transfer reactions [125].

These and similar examples have recently attracted a lot of attention in the theoretical community [126-136] although, from the very beginning, the DH forms were announced as the way to go in the future $[137,138]$; more discussion can be found in the series of last annual reviews highlighting achievements in computational organic chemistry [139-141].

We have recently studied (cc-pVTZ basis set) the stability of the polyhedrane family of hydrocarbons $(\mathrm{CH})_{2 n}(n=6,8,10,12$; see Figure 3$)$ as an example of the subtle interplay found between intra-molecular short-, medium-, and long-range (in the weak overlap region) physical effects when increasing the size of a system. The binding energy of both isomers, cage-like and planar, is calculated by the expression $\Delta E=\frac{1}{n} E\left[(\mathrm{CH})_{2 n}\right]-E\left[\left(\mathrm{C}_{2} \mathrm{H}_{2}\right)\right]$ and demands the greatest possible accuracy [142]. Figure 4 documents how pure and hybrid models accumulate large errors with respect to benchmark results, contrarily to DH functionals, although these are largely reduced in all cases upon adding a dispersion correction. However, if one closely inspects the values for isomerization energies, the trend is now more pronounced in favour of the DH forms: MUE of 33, 21, 8, and $2 \mathrm{kcal} / \mathrm{mol}$ are obtained for BLYP, B3LYP, B2-PLYP, and B2GP-PLYP, respectively, which further reduces upon addition of the -D2 dispersion correction to reach final values of $24,12,4$, and $2 \mathrm{kcal} / \mathrm{mol}$. More examples of this robust performance can 
be found for other challenging systems which are known to be a longstanding problem for standard functionals, such as: (i) the hydrogenation reaction of [2.2]paracyclophane to $p$-xylene [143]; (ii) the dimerization of tetramethylethene to yield the covalently bound octamethyl-cyclobutane [144,145]; (iii) the photodimerization of anthracene [146-148]; and (iv) the interconversion between two equivalent bowl forms of coroannulene [149]. In all these studied cases, B2-PLYP or PBE0-DH unambiguously outperform all older methods $[59,90,150]$.

However, even the DH forms can not cope with all intriguing effects arising at the quantum scale. As a matter of illustration, the energy barrier for the auto-isomerization of cyclobutadiene is known to be a longstanding problem [151-154]. The interconversion between two iso-energetic (rectangular) forms passes through a (square) transition state having degenerate frontier orbitals, see Figure 5, which would thus need a multiconfigurational treatment or a more generalised treatment than that provided by current methods [155-157]. Actually, the results provided by B2-PLYP (PBE0-DH), with the def2-QZVP basis set, severely overestimate that energy barrier: $23.5(25.2) \mathrm{kcal} / \mathrm{mol}$, respectively, to be compared against sophisticated multiconfigurational MR-BWCCSD $(\mathrm{T})$ results $(7.0 \mathrm{kcal} / \mathrm{mol}$, taken from Ref. [158]). Note also that these results compare favourably to those provided by CCSD [159], which scales an order of magnitude higher than DH. We have also investigated within this context the strong correlation effects present in a chain exclusively composed of many hydrogen atoms, serving as a model for understanding metallic to insulating transitions in real systems upon concerted bond stretching [160]. Simple as it seems, this model system can be considered as specially stringent and demanding for electronic struc- 
ture theories [161-164]. Actually, Figure 6 presents the potential energy curve for a simultaneous stretching of all bonds; the DH forms behave poorly along the dissociation path compared with nearly-exact results, as it also happens with all other applied DFT- or MP2-based methods. The performance of DH forms has been recently evaluated [165] for the prediction of enthalpies of formation of a large set of molecules containing $3 \mathrm{~d}$ transition metals. In this case, the mPW2-PLYP functional provided the lowest MUE among all tested method, including B2-PLYP, B2GP-PLYP, and B3LYP among others. Nonetheless, and in line with the above conclusion, if the global set is decomposed into two subsets for species having significant Multi-Reference (MR) character or Not (NMR), the MUE of all density functionals consistently decreased on going from the MR to the NMR set.

The importance of processes involving electronically excited states, and the dispersion of results that one can again face depending on the theoretical level selected, motivated us to include a brief description of the performance of DH forms for these phenomena. Again, a set of benchmark results is normally used to evaluate the performance of methods on a equal footing; which, however, complicates the things further due to environmental effects [166]. In this regard, computation of vertical singlet excitation energies for a set of all-organic aliphatic, aromatic and heteroaromatic chromophores (Thiel's database [167]) shows that the smallest deviations (less than $0.2 \mathrm{eV}$ ) are obtained by B2-PLYP [168]. Furthermore, for increasingly longer cyanine dyes, this model also behaves in an excellent way [169] with deviations again in the order of $0.1-0.2 \mathrm{eV}$, and thus admittedly close to the greatest conceivable accuracy for a TD-DFT calculation. Note that applications to excited-states are still scarce to reach final conclusions although, however, the existing re- 
sults are very promising yet needing additional technicalities too.

The study of magnetism in graphene nanoribbons or graphene nanoflakes, materials with envisioned applications in spintronic devices, using DH forms (B2-PLYP) has allowed to conclude that incorporation of the MP2-type contribution suffices to quenches the symmetry-breaking of spin densities $[170,171]$. This is not a trivial conclusion, which have contributed lately to the vivid debate in literature about half-metallicity of graphene, since a non-vanishing singlet-triplet energy gap is predicted at all other DFT levels, after the corresponding initial polarisation of spin-densities yielding antiferromagnetic systems, and only high-level $\operatorname{CCSD}(\mathrm{T})$ calculations reverse the situation indicating a vanishing singlet-triplet energy gap [172]. Another recent application for the field of materials science is the study of $n$-channel organic semiconductors based on N-heteroquinones [173], see Figure 7. The link to charge mobility values in the diffusive regime, where the charge carriers migrate across the molecular crystal through sequential (hopping) chargetransfer events involving a pair of neighbouring molecules, needs to access to the (sometimes unknown) dimer geometry. The applied DH form succeeded in providing the energy barrier for the self-exchange charge-transfer reaction, $\mathrm{M}+\mathrm{M}^{--} \longrightarrow \mathrm{M}^{--}+\mathrm{M}$, together with the intermolecular distance and the asymmetrical displacement of the slipped $\pi$-stacked molecules. This successful application of dispersion-corrected DH forms also extends to intramolecular processes, taken a set of (oligo)dibenzofulvenes (see Figure 8) as example [174], after predicting with high accuracy the face-to-face distance and orientation of the $\pi$-stacked constituting fluorene units. It thus seems that these methods can be robustly applied to nanostructured materials too, specially when the target properties are influenced by the supramolecular 
organisation of the samples.

Other recent applications, the (necessarily limited and biased) following selection tries only to show the wide and rich range of systems being currently afforded, include the modelling of ionic liquids [175], gas adsorption in metalorganic frameworks $[176,177]$, isomerism in monosaccharides [178], conformational analysis [179,180], nonlinear optical responses [181], magnetic couplings in organometallics [182], enzymatic catalysis [183,184], electron paramagnetic resonance hyperfine coupling tensors [185], inclusion complexes and nanoencapsulation [186], electronic circular dichroism [187], organometallic complexes of graphene [188], interfacial chemistry [189], photosynthetic water oxidation [190], hyperpolarizability of pushpull systems [191], lightharvesting complexes [192], adsorbate-zeolite interactions [193], or harmonic and anharmonic vibrational frequency calculations [194], among others.

\section{Conclusions}

Double-hybrid density functionals have unquestionably entered into the field of theoretical chemistry purposefully. They are effectively contributing to cutting-edge research since they are in the way of providing a right answer for the right reason, and can thus potentially push forward further projects. As already happens with older functionals, there are few flavors for their development leading to a variety of final expressions too. However, if they want to keep as a cornerstone, simplicity and elegance should be also concomitantly preserved. The responsibility of users for their choice is even higher: they are even less black-box tools than older hybrids and need additional technicalities. There still exists open issues, as in every field. 
We have already listed few of them in an attempt to help in further interpretation of the results. A simple measure of the great success of DH forms relies in the fact that all these open issues are expected to affect only marginally the results. We also remind that these methods are all based on a single-reference wavefunction, in the case of ground-states, or on a (mostly) single-reference/single-excitation treatment, in the case of excited-states, and thus might fail in situations where a breakdown of even sophisticated singlereference $a b$ initio treatments, as $\operatorname{CCSD}(\mathrm{T})$ for instance, is expected. Overall, to be always on the safer side due to the possible interplay between subtle physical effects, we recommend to progressively discard older functionals for routine use in the broad field of Computational Chemistry, giving thus to double-hybrid functionals larger and larger prominence if the computational cost does not become a serious bottleneck.

\section{Acknowledgements}

The work in Alicante is supported by the "Ministerio de Educación y Ciencia" of Spain and the "European Regional Development Fund" through project CTQ2011-27253. The work in Paris is supported by the "Agence Nationale de la Recherche" of France through project DinfDNT ANR 2010 BLANC No. 042502 and by Sanofi-Aventis.

\section{References}

[1] J. C. Slater, Phys. Rev., 1951, 81, 385.

[2] A. D. Becke, J. Chem. Phys., 1993, 98, 1372.

[3] A. D. Becke, J. Chem. Phys., 1993, 98, 5648. 
[4] J. P. Perdew, M. Ernzerhof, and K. Burke, J. Chem. Phys., 1996, 105, 9982.

[5] C. Adamo and V. Barone, Chem. Phys. Lett., 1997, 274, 242.

[6] M. Ernzerhof and G. E. Scuseria, J. Chem. Phys., 1999, 110, 5029.

[7] C. Adamo and V. Barone, J. Chem. Phys., 1999, 110, 6158.

[8] C. Malloy, "The Cleanest line" in Patagonia Surf 2006, catalog of Patagonia Inc, Reno, Nevada.

[9] B. Hammer, L. B. Hansen, and J. K. Nørskov, Phys. Rev. B, 1999, 11, 7413.

[10] J. Toulouse, A. Savin, and C. Adamo, J. Chem. Phys., 2002, 117, 10465.

[11] J. Tao, J. P. Perdew, V. N. Staroverov, and G. E. Scuseria, Phys. Rev. Lett., 2003, 91, 146401.

[12] S. Ragot and P. Cortona, J. Chem. Phys., 2004, 121, 7671.

[13] V. Tognetti, P. Cortona, and C. Adamo, J. Chem. Phys., 2008, 128, 034101.

[14] J. P. Perdew, K. Burke, and M. Ernzerhof, Phys. Rev. Lett., 1996, 77, 3865

[15] A. D. Becke, Phys. Rev. A, 1988, 38, 3098.

[16] C. Lee, W. Yang and R. G. Parr, Phys. Rev. B, 1988, 37, 785.

[17] J. Harris, Phys. Rev. A, 1984, 29, 1648.

[18] V. Barone and C. Adamo, Chem. Phys. Lett., 1994, 224, 432. 
[19] P. J. Stephens, F. J. Devlin, C. F. Chabalowski and M.J Frisch, J. Phys. Chem., 1994, 98, 11623.

[20] P. Mori-Sánchez, A. J. Cohen, and W. Yang, J. Chem. Phys., 2006, 124, 091102.

[21] M. J. G. Peach, A. M. Teale, and D. J. Tozer, J. Chem. Phys., 2007, 126, 244104.

[22] A. J. Cohen, P. Mori-Sánchez, and W. Yang, J. Chem. Phys., 2007, 127, 034101.

[23] M. J. G. Peach, A. M. Miller, A. M. Teale, and D. J. Tozer, J. Chem. Phys., 2008, 129, 064105.

[24] I. Ciofini, C. Adamo, and V. Barone, J. Chem. Phys., 2004, 121, 6710.

[25] M. P. Braun, H. Braun, N. Hojdis, and M. Bühl, J. Chem. Theory Comput., 2007, 3, 2234.

[26] D. Jacquemin, V. Wathelet, E. A. Perpète, and C. Adamo, J. Chem. Theory Comput., 2009, 5, 2420.

[27] X. Wu, A. Selloni, and R. Car, Phys. Rev. B, 2009, 79, 085102.

[28] C. Zhang, D. Donadio, F. Gygi, and G. Galli, J. Chem. Theory Comput., 2011, 7, 1443.

[29] P. Cortona, J. Chem. Phys., 2012, 136, 086101.

[30] C. A. Guido, E. Brémond, C. Adamo, and P. Cortona, J. Chem. Phys., 2013, 138, 021104.

[31] M. Ernzerhof, Chem. Phys. Lett., 1996, 263, 499. 
[32] K. Sharkas, J. Toulouse, and A. Savin, J. Chem. Phys., 2011, 134, 024106.

[33] M. Levy and J. P. Perdew, Phys. Rev. A, 1985, 32, 2010.

[34] J. Toulouse, K. Sharkas, E. Brémond, and C. Adamo, J. Chem. Phys., 2011, 135, 101102.

[35] S. Grimme, J. Chem. Phys., 2006, 124, 034108.

[36] C. Fiolhais, F. Nogueira, and M. A. Marques, in A primer in Density Functional Theory, Vol. 620, Springer, 2003.

[37] E. Brémond and C. Adamo, J. Chem. Phys., 2011, 135, 024106.

[38] T. Leininger, H. Stoll, H.-J. Werner, and A. Savin, Chem. Phys. Lett., 1997, 275, 151.

[39] H. Ikura, T. Tsuneda, T. Yanai, and K. Hirao, J. Chem. Phys., 2001, 115,3540 .

[40] J. G. Angyan, I. C. Gerber, A. Savin, and J. Toulouse, Phys. Rev. A, 2005, 72, 012510.

[41] E. Fromager, F. Réal, P. Wahlin, U. Wahlgren, H. J. Aa. Jensen, J. Chem. Phys., 2009, 131, 054107.

[42] E. Goll, H.-J. Werner, and H. Stoll, Phys. Chem. Chem. Phys., 2005, 7, 3917.

[43] E. Fromager, J. Toulouse, and H. J. Aa. Jensen, J. Chem. Phys., 2007, 126, 074111. 
[44] Y. Zhang, X. Xu, and W. A. Goddard III, Proc. Natl. Acad. Sci. USA, 2009, 106, 4963.

[45] Y. Zhang, J. Wu, X. Xu, Chem. Commun., 2010, 46, 3057.

[46] J.-D. Chai and S.-P. Mao, Chem. Phys. Lett., 2012, 538, 121.

[47] Y. Zhao, B. J. Lynch, and D. G. Truhlar, J. Phys. Chem. A, 2004, 108, 4786.

[48] Y. Zhao, B. J. Lynch, and D. G. Truhlar, Phys. Chem. Chem. Phys., 2005, 7, 43.

[49] J. C. Sancho-García, J. Phys. Chem. A, 2005, 109, 3470.

[50] Y. Zhao and D. G. Truhlar, J. Phys. Chem. A, 2005, 109, 4209.

[51] J. C. Sancho-García, J. Chem. Phys., 2006, 124, 124122.

[52] E. K. U. Gross, M. Petersilka, and T. Grabo, in Density Functional Methods in Chemistry, edited by T. Ziegler (ACS Symposium Series, Washington, 1996).

[53] T. Schwabe and S. Grimme, Phys. Chem. Chem. Phys., 2006, 8, 4398.

[54] F. Neese, T. Schwabe, and S. Grimme, J. Chem. Phys., 2007, 126, 124115 .

[55] K. Eichkorn, O. Trutler, H. Öhm, M. Häser, and R. Ahlrichs, Chem. Phys. Lett., 1995, 240, 283.

[56] F. Neese, F. Wennmohs, A. Hansen, and U. Becker, Chem. Phys., 2009, 356, 98 . 
[57] A. Tarnopolsky, A. Karton, R. Sertchook, D. Vuzman, and Jan M. L. Martin, J. Phys. Chem. A, 2008, 112, 3.

[58] A. Karton, A. Tarnopolsky, J.-F. Lamère, G. C. Schatz and, Jan M. L. Martin, J. Phys. Chem. A, 2008, 112, 12868.

[59] J. C. Sancho-García and A. J. Pérez-Jiménez, J. Chem. Phys., 2009, 131, 084108.

[60] D. C. Graham, A. S. Menon, L. Goerigk, S. Grimme, and L. Radom, J. Phys. Chem. A, 2009, 113, 9861.

[61] S. Kozuch, D. Gruzman, and Jan M. L. Martin, J. Phys. Chem. C, 2010, 114, 20801.

[62] S. Kozuch and Jan M. L. Martin, Phys. Chem. Chem. Phys., 2011, 13, 20104.

[63] B. Chan and L. Radom, J. Chem. Theory Comput., 2011, 7, 2852.

[64] Y. Zhang, X. Xu, Y. Jung, and W. A. Goddard III, Proc. Natl. Acad. Sci. USA, 2011, 108, 19896.

[65] L. Goerigk and S. Grimme, J. Chem. Theory Comput., 2011, 7, 291.

[66] K. Sharkas, J. Toulouse, and A. Savin, J. Chem. Phys., 2011, 134, 064113.

[67] A. Mohajeri and M. Alipour, J. Chem. Phys., 2012, 136, 124111.

[68] I. Y. Zhang, N. Q. Su, E. Brémond, C. Adamo, and X. Xu, J. Chem. Phys., 2012, 136, 174103.

[69] E. Fromager, J. Chem. Phys., 2012, 135, 244106. 
[70] S. Grimme, J. Chem. Phys., 2003, 118, 9095.

[71] D. K. W. Mok, R. Neumman, and N. C. Handy, J. Phys. Chem., 1996, 100,6225 .

[72] J. C. Sancho-García and F. Moscardó, J. Chem. Phys., 2003, 118, 1054.

[73] T. Benighaus, R. A. DiStasio Jr., R. C. Lochan, J.-D. Chai, and M. Head-Gordon, J. Phys. Chem. A, 2008, 112, 2702.

[74] J.-D. Chai and M. Head-Gordon, J. Chem. Phys., 2009, 131, 174105.

[75] I. Y. Zhang and X. Xu, J. Phys. Chem. Lett., 2013, 4, 1669.

[76] J. F. Dobson and T. Gould, J. Phys.: Condens. Matter, 2012, 24, 073201.

[77] O. A. Vydrov and T. Van Voorhis, J. Chem. Phys., 2010, 133, 244103.

[78] D. Cremer, WIREs Comput. Mol. Sci., 2011, 1, 509.

[79] Y. Zhao, N. E. Schultz, and D. G. Truhlar, J. Chem. Theory Comput., 2006, 2, 364 .

[80] A. Vazquez-Mayagoitia, C. D. Sherrill, E. Apra, and B. G. Sumper, J. Chem. Theory Comput., 2010, 6, 727.

[81] D. C. Langreth and J. P. Perdew, Phys. Rev. B, 1977, 15, 2884.

[82] H. Eshuis, J. E. Bates, and F. Furche, Theor. Chem. Acc., 2012, 131, 1084 .

[83] S. Grimme, J. Comput. Chem., 2006, 27, 1787.

[84] S. Grimme, J. Antony, S. Ehrlich and H. Krieg, J. Chem. Phys., 2010, 132, 154104 . 
[85] S. Grimme, WIREs Comput. Mol. Sci., 2011, 1, 211.

[86] Y. Liu and W. A. Goddard III, Mater. Trans., 2009, 50, 1664.

[87] P. Jurečka, J. Sponer, J. Černy, and P. Hobza, Phys. Chem. Chem. Phys., 2006, 8, 1985.

[88] T. Tatakani, E. G. Hohenstein, M. Malagoli, M. S. Marshall, and C. D. Sherrill, J. Chem. Phys., 2010, 132, 144104.

[89] M. S. Marshall, L. A. Burns, and C. D. Sherrill, J. Chem. Phys., 2011, 135, 194102.

[90] J. C. Sancho-García, Chem. Phys. Lett., 2012, 535, 136.

[91] T. Schwabe and S. Grimme, Phys. Chem. Chem. Phys., 2007, 9, 3397.

[92] T. Schwabe, R. Huenerbein, and S. Grimme, SYNLETT, 2010, 10, 1431.

[93] L. Goerigk and S. Grimme, in Modeling of Molecular Properties, edited by P. Comba (Wiley-VCH, Verlag, 2011).

[94] J. Klimeš and A. Michaelides, J. Chem. Phys., 2012, 137, 120901.

[95] S. Ehrlich, H. Moellmann, and S. Grimme, Acc. Chem. Res., 2013, 45, 916.

[96] S. Grimme and F. Neese, J. Chem. Phys., 2007, 127, 154116.

[97] M. Head-Gordon, R. J. Rico, M. Oumi, and T. J. Lee, Chem. Phys. Lett., 1994, 219, 21.

[98] L. Goerigk, J. Moellmann, and S. Grimme, Phys. Chem. Chem. Phys., 2009, 11, 4611. 
[99] L. Goerigk and S. Grimme, J. Chem. Phys., 2010, 132, 184103.

[100] S. Grimme, L. Goerigk, and R. F. Fink, WIREs Comput. Mol. Sci., $2012, \mathbf{2}, 886$.

[101] L. Goerigk and S. Grimme, J. Chem. Theory Comput., 2011, 7, 3272.

[102] J. Almlöf, Chem. Phys. Lett., 1991, 181, 319.

[103] Y. Jung, R. C. Lochan, A. D. Dutoi, and M. Head-Gordon, J. Chem. Phys., 2004, 121, 9793.

[104] A. Karton and Jan M. L. Martin, J. Chem. Phys., 2011, 135, 144119.

[105] A. Halkier, T. Helgaker, P. Jorgensen, W. Klopper, H. Koch, J. Olsen, and A. K. Wilson, Chem. Phys. Lett., 1998, 286, 243.

[106] S. Parthiban and Jan M. L. Martin, J. Chem. Phys., 2001, 114, 6014.

[107] I. Y. Zhang and X. Xu, Int. Rev. Phys. Chem., 2011, 30, 115.

[108] I. Y. Zhang, Y. Luo, and X. Xu, J. Chem. Phys., 2010, 132, 194105.

[109] I. Y. Zhang, Y. Luo, and X. Xu, J. Chem. Phys., 2010, 133, 104105.

[110] D. W. Schwenke, J. Chem. Phys., 2005, 122, 014107.

[111] Y.-Y. Chuang and S.-M. Chen, J. Comput. Chem., 2011, 32, 1671.

[112] Y.-Y. Chuang and S.-M. Chen, J. Chin. Chem. Soc., 2012, 59, 1094.

[113] P. Mori-Sánchez, A. J. Cohen, and W. Yang, J. Chem. Phys., 2006, 125, 201102.

[114] H. Lee Woodcock, H. F. Schaefer III, and P. R. Schreiner, J. Phys. Chem. A, 2002, 106, 11923. 
[115] I. Ciofini, H. Chermette, and C. Adamo, Chem. Phys. Lett., 2003, 380, 12.

[116] I. Ciofini, C. Adamo, and H. Chermette, J. Chem. Phys., 2005, 123, 121102.

[117] M. Lundberg and P. E. M. Siegbahn, J. Chem. Phys., 2005, 122, 224103.

[118] J. C. Sancho-García and A. J. Pérez-Jiménez, J. Phys. Chem. A, 2008, $112,10325$.

[119] A. J. Cohen, P. Mori-Sánchez, and W. Yang, Chem. Rev., 2012, 112, 289.

[120] L. Goerigk and S. Grimme, Phys. Chem. Chem. Phys., 2011, 13, 6670.

[121] L. Goerigk and S. Grimme, J. Chem. Theory Comput, 2010, 6, 107.

[122] S. Luo, Y. Zhao, and D. G. Truhlar, Phys. Chem. Chem. Phys., 2011, 13, 13683.

[123] H. Krieg and S. Grimme, Mol. Phys., 2010, 108, 2655.

[124] S. Grimme, Org. Lett., 2010, 12, 1440.

[125] G. F. Mangiatordi, E. Brémond, and C. Adamo, J. Chem. Theory Comput, 2012, 8, 3082.

[126] M. D. Wodrich, C. Corminboeuf, and P. v. R. Schleyer, Org. Lett., $2006,8,3631$.

[127] P. R. Schreiner, A. A. Fokin, R. A. Pascal Jr., and A. de Meijere, Org. Lett., 2006, 8, 3635 . 
[128] P. R. Schreiner, Angew. Chem. Int. Ed., 2007, 46, 4217.

[129] M. D. Wodrich, C. Corminboeuf, P. R. Schreiner, A. A. Fokin, and P. v. R. Schleyer, Org. Lett., 2007, 9, 1851.

[130] S. Grimme, M. Steinmetz, and M. Korth, J. Org. Chem., 2007, 72, 2118.

[131] M. D. Wodrich, D. F. Jana, P. v. R. Schleyer, and C. Corminboeuf, J. Phys. Chem. A, 2008, 112, 12495.

[132] A. Karton, D. Gruzman, and Jan M. L. Martin, J. Phys. Chem. A, 2009, 113, 8434 .

[133] D. R. B. Brittain, C. Y. Lin, A. T. B. Gilbert, E. I. Izgorodina, P. M. W. Gill, and M. L. Coote, Phys. Chem. Chem. Phys., 2009, 11, 1138.

[134] S. N. Steinmann, M. D. Wodrich, and C. Corminboeuf, Theor. Chem. Acc., 2010, 127, 429.

[135] J.-W. Song, T. Tsuneda, T. Sato, and K. Hirao, Org. Lett., 2010, 12, 1440.

[136] D. H. Ess, S. liu, and F. De Proft, J. Phys. Chem. A, 2010, 114, 12952.

[137] S. Grimme, Angew. Chem. Int. Ed., 2006, 45, 4460.

[138] S. Grimme, M. Steinmetz, and M. Korth, J. Chem. Theory Comput., 2007, 3, 42 .

[139] S. M. Bachrach, Annu. Rep. Prog. Chem., Sect. B: Org. Chem., 2012, 108,334 . 
[140] S. M. Bachrach, Annu. Rep. Prog. Chem., Sect. B: Org. Chem., 2011, 107,349 .

[141] S. M. Bachrach, Annu. Rep. Prog. Chem., Sect. B: Org. Chem., 2010, $106,407$.

[142] J. C. Sancho-García, J. Chem. Theory Comput, 2011, 7, 2761.

[143] S. Grimme, Chem. Eur. J, 2004, 10, 3423.

[144] S. Grimme, Angew. Chem. Int. Ed., 2006, 45, 625.

[145] J. Quenneville and T. C. Germann, J. Chem. Phys., 2009, 131, 024313.

[146] S. Grimme, Angew. Chem. Int. Ed., 2006, 45, 625.

[147] G. I. Csonka, A. Ruzsinszky, J. P. Perdew, and S. Grimme, J. Chem. Theory Comput., 2008, 4, 888.

[148] F. Neese, F. Wennmohs, and A. Hansen, J. Chem. Phys., 2009, 130, 114108.

[149] R. Peverati and K. K. Baldridge, J. Chem. Theory Comput., 2008, 4, (2008).

[150] J. C. Sancho-García, J. Chem. Phys., 2011, 134, 234102.

[151] A. Balková and R. J. Barlett, J. Chem. Phys., 1994, 101, 8972.

[152] J. C. Sancho-García, J. Pittner, P. Čársky, and I. Hubač, J. Chem. Phys., 2000, 112, 8785.

[153] K. Bhaskaran-Nair, O. Demel, and J. Pittner, J. Chem. Phys., 2008, 129, 184105. 
[154] U. S. Mahapatra, S. Chattopadhyay, and R. K. Chaudhuri, J. Comput. Chem., 2011, 32, 325.

[155] M. Filatov and S. Shaik, J. Chem. Phys., 1999, 110, 116.

[156] S. Grimme and M. Waletzke, J. Chem. Phys., 1999, 111, 5645.

[157] J. C. Sancho-García, A. J. Pérez-Jiménez, and F. Moscardó, Chem. Phys. Lett., 2000, 317, 245.

[158] O. Demel and J. Pittner, J. Chem. Phys., 2006, 124, 144112.

[159] M. Musial, A. Perera, and R. J. Barlett, J. Chem. Phys., 2011, 134, 114108.

[160] T. Giamarchi, Chem. Rev., 2007, 104, 5037.

[161] J. Hachmann, W. Cardoen, and G. K.-L. Chan, J. Chem. Phys., 2006, 125, 144101.

[162] D. A. Mazziotti, Acc. Chem. Res., 2006, 39, 207.

[163] T. Tsuchimochi and G. E. Scuseria, J. Chem. Phys., 2009, 131, 121102.

[164] J. C. Sancho-García, Chem. Phys. Lett., 2011, 511, 172.

[165] W. Jiang, M. L. Laury, M. Powell, and A. K. Wilson, J. Chem. Theory Comput., 2012, 8, 4102.

[166] D. Jacquemin, E. A. Perpète, I. Ciofini, and C. Adamo, Acc. Chem. Res., 2009, 42, 326.

[167] M. R. Silva-Junior, M. Schreiber, S. P. A. Sauer, and W. Thiel, J. Chem. Phys., 2010, 133, 174318. 
[168] D. Jacquemin, B. Mennucci, and C. Adamo, Phys. Chem. Chem. Phys., 2011, 13, 16987.

[169] R. Send, O. Valsson, and C. Filippi, J. Chem. Theory Comput., 2011, 7,444 .

[170] S. Rayne and K. Forest, Comput. Theor. Chem., 2011, 977, 163.

[171] B. Hagjató and M. S. Deleuze, Chem. Phys. Lett., 2012, 553, 6.

[172] M. Huzak, M. S. Deleuze, and B. Hagjató, J. Chem. Phys., 2011, 134, 104704 .

[173] J. C. Sancho-García, J. Chem. Phys., 2012, 136, 174703.

[174] J. C. Sancho-García, Theor. Chem. Acc., 2010, 127, 605.

[175] Y.-H. Tian, G. S. Goff, W. H. Runde, and E. R. Batista, J. Phys. Chem. B, 2012, 116, 11943.

[176] W. Li, S. Grimme, H. Krieg, J. Möllmann, and J. Zhang, J. Phys. Chem. C, 2012, 116, 8865 .

[177] L. Chen, C. A. Morrison, and T. Düren, J. Phys. Chem. C, 2012, 116, 18899.

[178] W. M. C. Sameera, and D. A. Pantazis, J. Chem. Theory Comput., $2012,8,2630$.

[179] G. I. Csonka and J. Kaminsky, J. Chem. Theory Comput., 2011, 7, 988.

[180] D. Seebach, U. Grošelj, W. B. Schweizer, S. Grimme, and C. MückLichtenfeld, Helv. Chim. Acta, 2010, 93, 1. 
[181] F. Castet and B. Champagne, J. Chem. Theory Comput., 2012, 8, 2044 .

[182] N. A. G. Bandeira and B. L. Guennic, J. Phys. Chem. A, 2012, 116, 3465 .

[183] K. Hotta, X. Chen, R. S. Paton, A. Minami, H. Li, K. Swaminathan, I. I. Mathews, K. Watanabe, H. Oikawa, K. N. Houk, and C.-Y. Kim, Nature, 2012, 483, 355.

[184] J. Garrec, P. Sautet, and P. Fleurat-Lessar, J. Phys. Chem. B, 2011, $\mathbf{1 1 5}, 8545$.

[185] H. D. Hedegard, J. Kongsted, and S. P. A. Sauer, Phys. Chem. Chem. Phys., 2012, 14, 10669.

[186] M. P. Waller, H. Kruse, C. Mück-Lichtenfeld, and S. Grimme, Chem. Soc. Rev., 2012, 41, 3119.

[187] I. Warnke and F. Furche, WIREs Comput. Mol. Sci., 2012, 2, 150.

[188] S. M. Avdoshenko, I. N. Ioffe, G. Cuniberti, L. Dunsch, and A. A. Popov, ACS Nano, 2011, 5, 9939 .

[189] J. Aragó, J. C. Sancho-García, E. Ortí, and D. Beljonne, J. Chem. Theory Comput., 2011, 7, 2068.

[190] P. E. M. Siegbahn, J. Photochem. Photobiol. B, 2011, 104, 94.

[191] M. de Wergifosse and B. Champagne, J. Chem. Phys., 2011, 134, 074113.

[192] C. König and J. Neugebauer, Phys. Chem. Chem. Phys., 2011, 13, 10475 . 
[193] P. Tongying and Y. Tantirungrotechai, J. Mol. Struct., 2010, 945, 85.

[194] M. Biczysko, P. Panek, G. Scalmani, J. Bloino, and V. Barone, J. Chem. Theory Comput., 2010, 6, 2115. 
- Table 1. Brief summary of existing double-hybrid functionals, presented in chronological order within each ad hoc established cathegory.

- Table 2. Values of the $s_{6}$ parameter for common double-hybrid functionals, and associated MUE (kcal/mol) for the S22 database of weakly interacting systems.

- Table 3. MUE (kcal/mol) for the complete GMTKN30 database. 

Table 1:

\begin{tabular}{|c|c|c|c|c|c|}
\hline Cathegory & Acronym & Year & $\mathrm{w}_{\mathrm{EXX}}$ & $\mathrm{w}_{\mathrm{PT} 2}{ }^{\mathrm{a}}$ & Reference \\
\hline \multirow[t]{2}{*}{ Parameter-free } & PBE0-DH & 2011 & 0.50 & 0.125 & {$[37]$} \\
\hline & PBE0-2 & 2012 & 0.79 & 0.50 & {$[46]$} \\
\hline \multirow[t]{20}{*}{ Empirical forms } & B2-PLYP & 2006 & 0.53 & 0.27 & {$[35]$} \\
\hline & mPW2-PLYP & 2006 & 0.55 & 0.25 & {$[53]$} \\
\hline & B2K-PLYP & 2008 & 0.72 & 0.42 & {$[57]$} \\
\hline & B2T-PLYP & 2008 & 0.60 & 0.31 & {$[57]$} \\
\hline & mPW2K-PLYP & 2008 & 0.72 & 0.42 & {$[57]$} \\
\hline & B2GP-PLYP & 2008 & 0.65 & 0.36 & {$[58]$} \\
\hline & B2 $\pi$-PLYP & 2009 & 0.60 & 0.27 & {$[59]$} \\
\hline & (U)B2-PLYP & 2009 & 0.62 & 0.35 & {$[60]$} \\
\hline & (RO)B2-PLYP & 2009 & 0.59 & 0.28 & {$[60]$} \\
\hline & XYG3 & 2009 & 0.80 & 0.32 & {$[44]$} \\
\hline & DSD-BLYP & 2010 & 0.70 & $(0.46,0.40)^{\mathrm{b}}$ & {$[61]$} \\
\hline & DSD-PBEP86 & 2011 & 0.68 & $(0.51,0.23)^{\mathrm{b}}$ & {$[62]$} \\
\hline & $\mathrm{DuT}$ & 2011 & 0.70 & $(0.47,0.36)^{\mathrm{b}}$ & {$[63]$} \\
\hline & XYGJ-OS & 2011 & 0.77 & $(0.44,0.0)^{\mathrm{b}}$ & {$[64]$} \\
\hline & PTPSS & 2011 & 0.50 & $(0.38,0.0)^{\mathrm{b}}$ & {$[65]$} \\
\hline & PWPB95 & 2011 & 0.50 & $(0.27,0.0)^{\mathrm{b}}$ & {$[65]$} \\
\hline & 1DH-BLYP & 2011 & 0.65 & 0.42 & {$[66]$} \\
\hline & LS1DH & 2011 & 0.75 & 0.42 & {$[34]$} \\
\hline & B2-PPW91 & 2012 & 0.20 & 0.10 & {$[67]$} \\
\hline & xDH-PBE0 & 2012 & 0.83 & 0.54 & {$[68]$} \\
\hline \multirow[t]{4}{*}{ Range-separated ${ }^{c}$} & B2-P3LYP & 2008 & 0.53 & 0.27 & {$[73]$} \\
\hline & B2-OS3LYP & 2008 & 0.53 & $(0.32,0.0)^{\mathrm{b}}$ & {$[73]$} \\
\hline & $\omega \mathrm{B} 97 \mathrm{X}-2$ & 2009 & 0.64 & $(0.45,0.53)^{\mathrm{b}}$ & {$[74]$} \\
\hline & lrc-XYG3 & 2013 & 0.80 & 0.32 & {$[75]$} \\
\hline
\end{tabular}

${ }^{\mathrm{a}}$ Note that fewer forms relaxes the constraint $\mathrm{w}_{\mathrm{PT} 2} E_{c}^{\mathrm{PT} 2}+\left(1-\mathrm{w}_{\mathrm{PT} 2}\right) E_{c}[\rho]$.

$\mathrm{b}$ The two values refers to opposite- and same-spin coefficients, respectively.

c Additionally, the value of the $\omega$ porameter defining the short- and longrange separation is needed (normally $\omega=0.2-0.3 \mathrm{Bohr}^{-1}$ ) 
Table 2:

\begin{tabular}{llll}
\hline Functional & $s_{6}$ & MUE & Reference \\
\hline PBE/TZVPP & 0.75 & 1.0 & {$[35]$} \\
PBE0/TZVPP & 0.70 & 1.0 & {$[58]$} \\
PBE0-DH/def2-QZVP & 0.47 & 0.4 & {$[90]$} \\
BLYP/TZVPP & 1.20 & 0.7 & {$[35]$} \\
B3LYP/TZVPP & 1.05 & 0.8 & {$[35]$} \\
B2-PLYP/TZVPP & 0.55 & 0.3 & {$[91]$} \\
B2T-PLYP/TZVPP & 0.48 & 0.4 & {$[58]$} \\
B2GP-PLYP/TZVPP & 0.40 & 0.4 & {$[58]$} \\
B2K-PLYP/TZVPP & 0.30 & 0.4 & {$[58]$} \\
\hline
\end{tabular}


Table 3:

\begin{tabular}{ll}
\hline Functional & WTMUE \\
\hline BLYP-D3 & 5.3 \\
B3LYP-D3 & 3.7 \\
B2-PLYP-D3 & 2.0 \\
B2GP-PLYP-D3 & 1.7 \\
\hline
\end{tabular}


- Figure 1. Chemical structures of the dye benchmark set. The hydrogen atoms and corresponding $\mathrm{C}-\mathrm{H}$ bonds have been omitted for clarity.

- Figure 2. Evolution of MUE for the SIE11 database as a function of the weight of exact-like exchange introduced into the double-hybrid functional.

- Figure 3. Representation of the molecular geometries of cage-like (top) and planar (bottom) $(\mathrm{CH})_{2 n}(\mathrm{n}=6,8,10,12)$ polyhedrane hydrocarbons.

- Figure 4. Evolution of MUE for the binding energy of cage-like (left) and planar (right) $(\mathrm{CH})_{2 n}(\mathrm{n}=6,8,10,12)$ polyhedrane hydrocarbons.

- Figure 5. Reaction of auto-isomerization between rectangular forms of cyclobutadiene. The hydrogen atoms and corresponding $\mathrm{C}-\mathrm{H}$ bonds have been omitted for clarity.

- Figure 6. Potential energy curves for the symmetric and concerted dissociation of a $\mathrm{H}_{50}$ chain.

- Figure 7. Chemical structures of 5,7,12,14-tetraaza-6,13-pentacenequinone (1) and 1,4,8,11-tetraaza-6,13-pentacenequinone (2). The hydrogen atoms and corresponding $\mathrm{C}-\mathrm{H}$ bonds have been omitted for clarity. 
- Figure 8. Chemical structures and nomenclature of (oligo)dibenzofulvene organic molecular semiconductors. The hydrogen atoms and corresponding $\mathrm{C}-\mathrm{H}$ bonds have been omitted for clarity. 
<smiles>O=c1cc(C(F)(F)F)c2cc3c4c(c2o1)CCCN4CCC3</smiles>

1<smiles></smiles>

$\mathrm{Et}_{2} \mathrm{~N}$<smiles>CCNc1ccc2c(c1)CC1=CC(=O)c3ccccc3C1=N2</smiles><smiles>Cn1c2ccccc2c2cc3ccccc3cc21</smiles>

9<smiles></smiles><smiles>O=C1c2ccc(F)cc2NC1C1Nc2cc(F)ccc2C1=O</smiles>

2

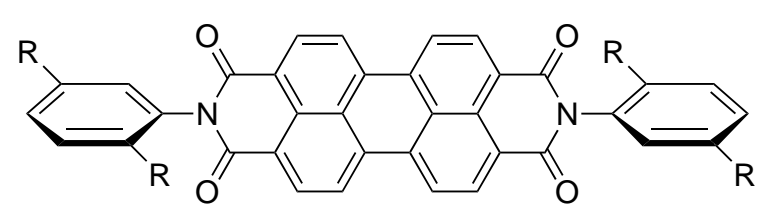

$R_{\text {theo }}=\mathrm{H}$
$\mathrm{R}_{\text {exp }}={ }^{\mathrm{t}} \mathrm{Bu}$

5<smiles>CNc1ccc2cc3ccc(=N)cc-3oc2c1</smiles><smiles></smiles>

10

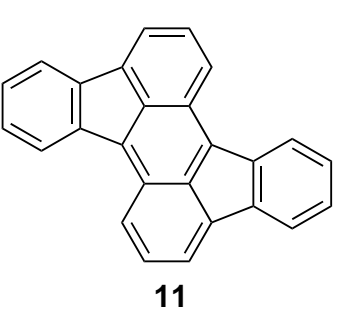

11

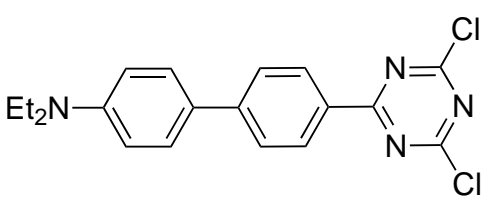

12

Figure 1. 


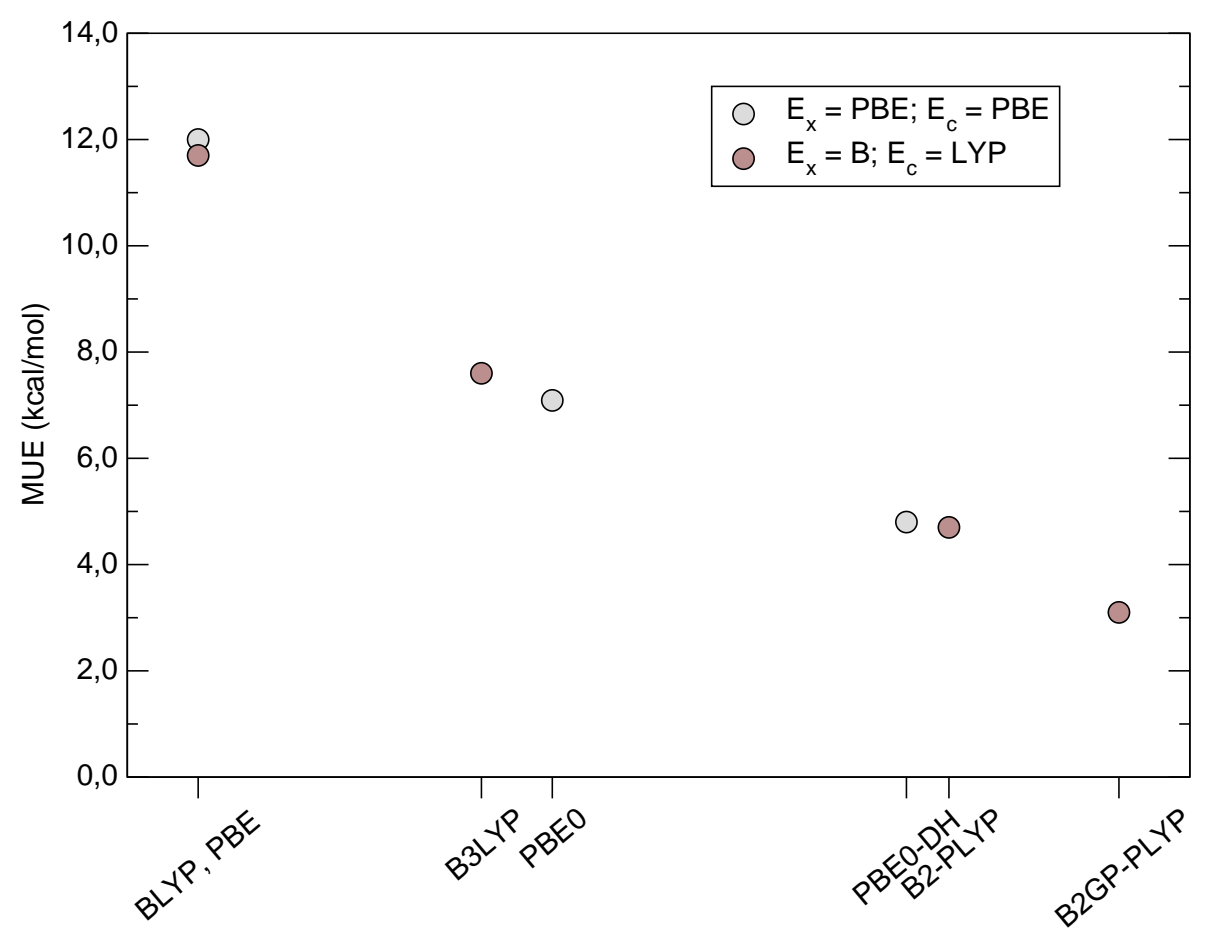

Figure 2. 

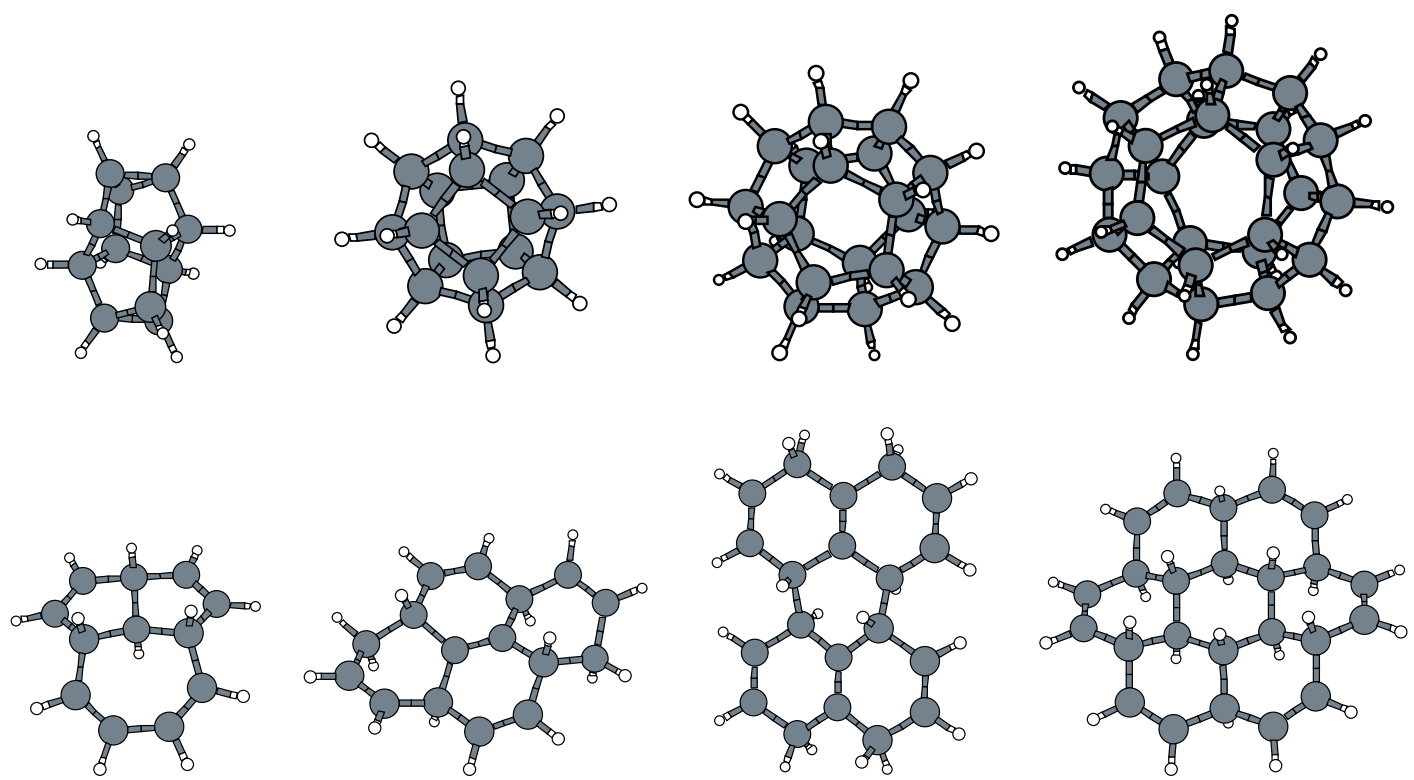

Figure 3. 

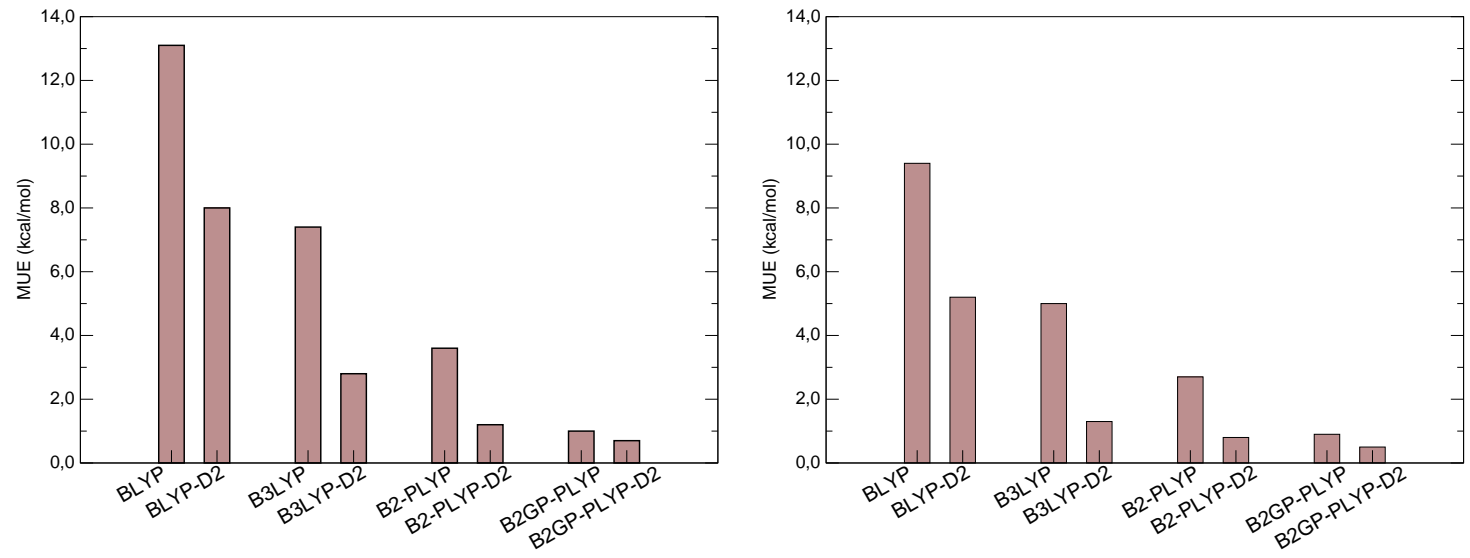

Figure 4. 


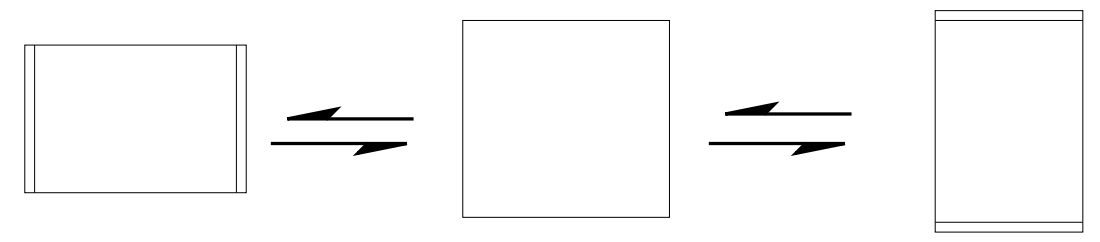

Figure 5. 


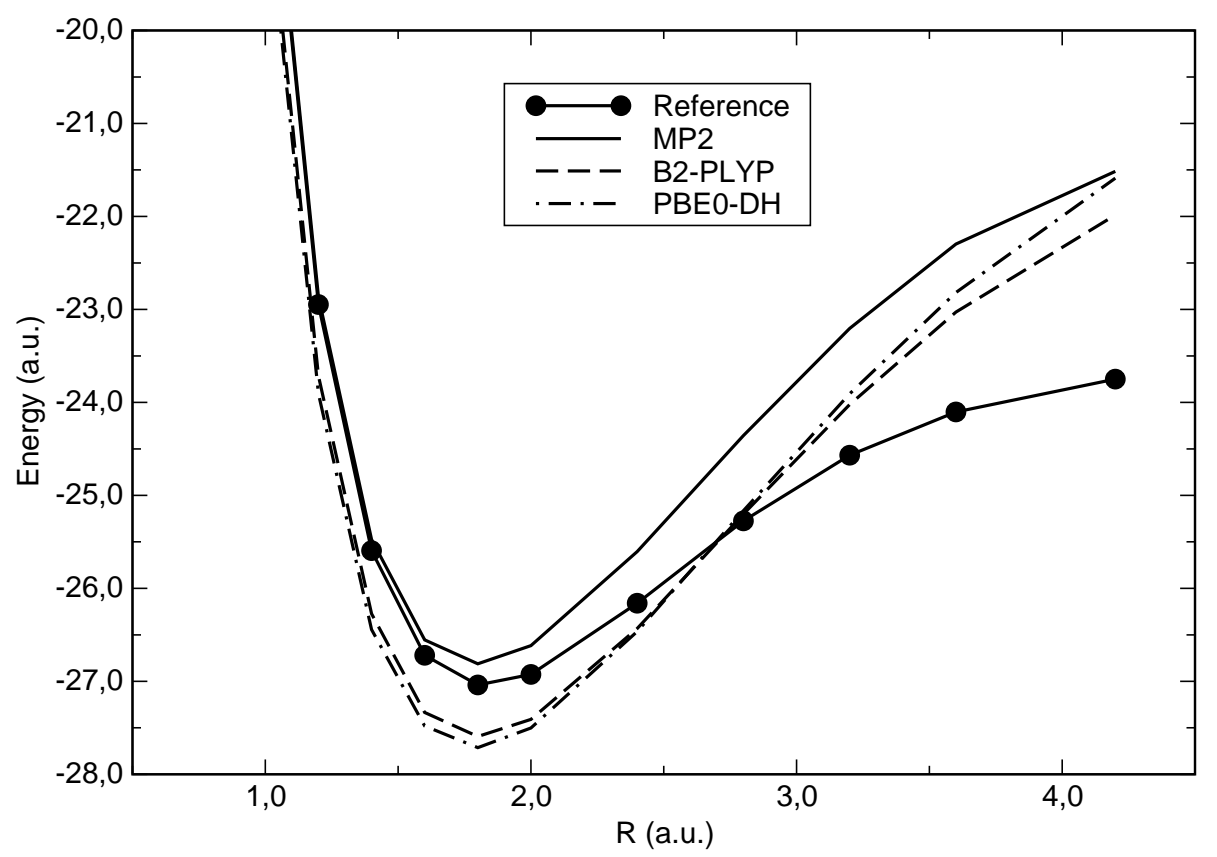

Figure 6. 

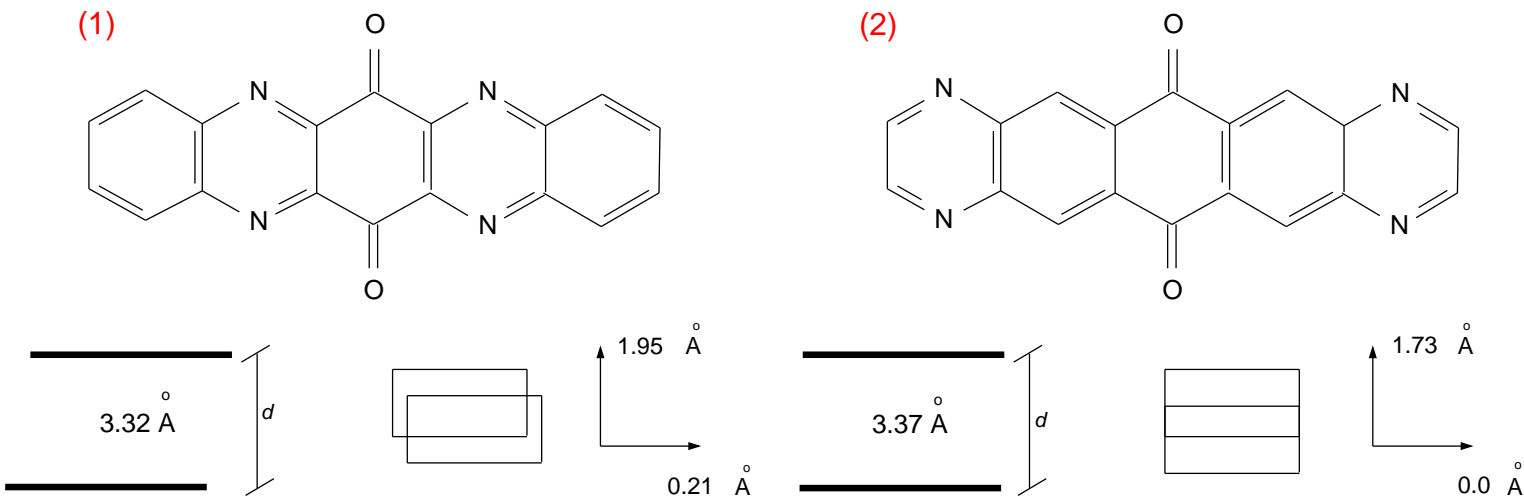

Figure 7. 


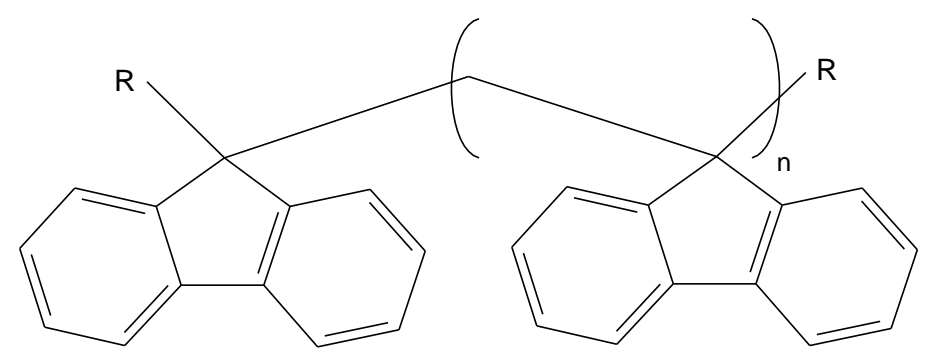

Figure 8. 